\title{
FATORES DE INFLUÊNCIA NA APRENDIZAGEM PERCEBIDA DOS ALUNOS DE CURSOS DE ADMINISTRAÇÃO
}

\author{
Tatiana Marceda Bach*; Silvana Anita Walter**; \\ José Roberto Frega***; Joer Maria Schvarcz Müller****
}

Recebido: 26 out. 2011

Aprovado: 05 dez. 2011

* Universidade Regional de Blumenau. Blumenau, SC, Brasil. Contato: tatibach@gmail.com

** Universidade Regional de Blumenau. Blumenau, SC, Brasil. Contato: silvanaanita.walter@gmail.com

*** Universidade Federal do Paraná. Curitiba, PR, Brasil. Contato: jose.frega@gmail.com

****Universidade Regional de Blumenau. Blumenau, SC, Brasil. Contato: joer@bombinhaspraia.com.br

Resumo: Este estudo objetivou analisar as dimensões que influenciam a aprendizagem dos alunos de dois cursos de graduação em administração de uma Instituição de Ensino Superior (IES) do sul do Brasil. Empregou-se a abordagem quantitativa, de caráter descritivo e, para a coleta dos dados, realizou-se um levantamento com 880 alunos, por meio do questionário estruturado de Walter (2006), que abrange 26 dimensões do ensino superior. Os dados foram analisados por meio de métodos de estatística descritiva, análise fatorial, Alfa de Cronbach e regressão linear multivariada. Os resultados indicam que a aprendizagem percebida é influenciada, principalmente, pelas dimensões satisfação, métodos de ensino, qualidade do ensino, empregabilidade, confiança nas pessoas da IES, atitude da coordenação e grade curricular, as quais apresentaram valor de significância $\mathrm{p}<0,001$. Já as dimensões autoeficácia e imagem social apresentaram uma significância $p<0,05$. As dimensões apontadas pelos alunos podem direcionar as ações da IES de forma a realçar os itens considerados importantes pelos alunos. Como contribuição teórica para a área de estudos, acredita-se que esta pesquisa possa auxiliar, por meio do número abrangente de dimensões, no entendimento de fatores que possam influenciar a aprendizagem de estudantes nas IES, visto que não se identificaram, na literatura, estudos desta natureza.

Palavras-chave: Aprendizagem percebida. Ensino superior. Fatores de influência.

\section{INFLUENCE FACTORS IN LEARNING \\ OF STUDENTS IN BUSINESS ADMINISTRATION PROGRAMS}

Abstract: This study aimed to analyze the dimensions that influence the learning of students in two undergraduate courses in administration of a Higher Education Institution (HEI) in southern Brazil. The study has a quantitative and descriptive approach, and for data collection was carried out a survey of 880 students, by means of a structured questionnaire Walter (2006), which covers 26 dimensions of higher education. The analysis was carried out using descriptive statistical methods, factor analysis, Cronbach's Alpha and multivariate linear regression. The results indicate that perceived learning is, mainly, influenced by the dimensions of satisfaction, teaching methods, teaching quality, employability, confidence in the people of HEI, coordination and attitude of the curriculum, which showed significance value $p<0.001$. Also, the dimensions of self-efficacy and social image showed a significance of $p<0.05$. The dimensions indicated by the students can direct the actions of the HEI, highlighting the items considered important by students. As a theoretical contribution to the field of study, it is believed that this research can help, through the extensive number of dimensions, the understanding of factors that may influence student learning in higher education institutions, since they were not identified in the literature, studies of this nature.

Key words: Perceived learning, Higher education. Influencing factors. 


\section{INTRODUÇÃO}

A partir da aprovação da Lei de Diretrizes e Bases (LDB) da Educação Superior de 1996, Lei $n^{\circ}$ 9.394, ocorreu um expressivo aumento no número de instituições de ensino superior (IES) e, consequentemente, o crescimento da competição entre as IES, principalmente no que se refere à conquista e à manutenção de alunos. Assim, as IES passaram a se interessar, ainda mais, pelo relacionamento com os alunos em curto, médio e longo prazo, bem como a se preocupar com esse relacionamento. Nesse contexto, a aprendizagem de estudantes, passou a ser alvo da atenção das instituições de ensino superior, por ser a razão fundamental de sua existência e o principal indicativo empregado para que os alunos percebam a qualidade de sua formação.

Entre os benefícios que uma positiva percepção da aprendizagem pelos alunos pode proporcionar às IES, pode-se citar a influência na qualidade pedagógica, o comprometimento (RODIE; KLEINE, 2000), a lealdade com a instituição (WALTER; TONTINI; DOMINGUES, 2006) e o retorno à instituição para atualização de conhecimentos (MARZO-NAVARRO; PEDRAJA-IGLESIAS; RIVERA-TORRES, 2005). Além disso, verificar a percepção que os estudantes possuem a respeito de seu aprendizado pode auxiliar as IES a desenvolverem estratégias de melhoria. Nesse sentido, Melo, Dutra e Oliveira (2001) destacam que as IES, cada vez mais, têm percebido que é importante considerar a percepção dos estudantes a respeito do ensino ofertado, pois isso pode indicar melhorias a serem realizadas. Reinert e Reinert (2005) acrescentam que os alunos necessitam ser considerados pela IES como parceiros em seu processo de ensino e de melhoria, visto que esse é o interesse em comum.

Diante do apresentado, percebe-se a relevância de avaliar a percepção dos alunos do Ensino Superior a respeito de sua aprendizagem. Apesar de existirem pesquisas voltadas à avaliação institucional por alunos, essas normalmente se concentram na avaliação de percepção da qualidade dos serviços (MELLO; DUTRA; OLIVEIRA, 2001; GONÇALVES FILHO; GUERRA; MOURA, 2003; LIN, 2006; GARCIA, 2007), satisfação (ALVES, 2003; GONÇALVES FILHO; GUERRA; MOURA, 2003; KAREMERA; REUBEN; SILLAH, 2003; WALTER, 2006; SOUZA; REINERT, 2010) e lealdade (HENNIG-THURAU; LANGER; HANSEN, 2001; GONÇALVES FILHO; GUERRA; MOURA, 2003; LIN, 2006; WALTER, 2006). Não se encontraram estudos especificamente sobre a aprendizagem percebida, abordando a influência que essa recebe de outras dimensões avaliadas pelos alunos do Ensino Superior. 
Visando a preencher essa lacuna, este estudo, sem ter a pretensão de esgotar o assunto, buscou responder à seguinte indagação: Qual a influência dos diferentes fatores relacionados ao curso de administração na aprendizagem percebida dos alunos? Assim, teve-se, por objetivo, analisar fatores que influenciam a aprendizagem percebida pelos alunos de dois cursos de graduação em administração de uma instituição de ensino do sul do país.

Este artigo está estruturado da seguinte forma: além desta primeira seção, na segunda, revisa-se a literatura enfocando estudos sobre dimensões relacionadas à aprendizagem de alunos no Ensino Superior; na terceira, apresenta-se o delineamento metodológico da pesquisa; na quarta, discutem-se os resultados; e, na quinta, expõem-se as considerações finais, implicações gerenciais e limitações do estudo.

\section{BASE TEÓRICA}

Nesta seção, apresentam-se pesquisas que evidenciam diferentes aspectos que podem influenciar a aprendizagem dos alunos de Ensino Superior, além de outros que a literatura identifica como aspectos que afetam a percepção da qualidade e da satisfação dos alunos, mas que também são investigados como possíveis influenciadores da aprendizagem dos estudantes.

Karemera, Reuben e Sillah (2003), por exemplo, perceberam que programas de desenvolvimento profissional e atividades extracurriculares, como estágios, viagens de estudo e intercâmbios, combinando as experiências de dentro e de fora da sala de aula, promovem a aprendizagem e o desenvolvimento dos alunos, estando essa relacionada a um melhor desempenho acadêmico. Os autores também verificaram que a escolha das matérias e a suficiência dos serviços de biblioteca estão associadas, significativamente, ao desempenho dos alunos.

Souza e Reinert (2010), por sua vez, identificaram que as categorias estrutura curricular e corpo docente são as que mais influenciam a satisfação ou a insatisfação dos estudantes do curso de administração. Além desses, os autores notaram outros dois fatores menos frequentes, mas que também afetam a satisfação ou insatisfação dos estudantes: ambiente social e infraestrutura.

Já Melo, Dutra e Oliveira (2001) destacam que o desempenho dos funcionários administrativos da instituição, a preocupação com a praticidade do conhecimento, o preparo dos professores e a integração da faculdade com executivos do mercado (visitas às empresas e palestras na faculdade) influenciam a percepção que os estudantes possuem a respeito da qualidade do ensino. $\mathrm{O}$ desempenho do professor durante as aulas, a forma como atende aos alunos, 
com clareza, facilidade, transparência e prontidão, se revelaram, na pesquisa desses autores, fundamentais para a qualidade do serviço educacional.

Os professores também tiveram destaque nos resultados do estudo de Garcia e Derisio (2007), no qual o construto que apresentou maior coeficiente de relacionamento com a qualidade foi capacitação do corpo docente. Em segundo lugar, ficou o construto relação entre professores e alunos; em terceiro, ambiente acadêmico; em quarto, aspectos tangíveis; em quinto, atendimento; e, por fim, relacionamento entre discentes. Por isso, os autores defendem que o principal aspecto a ser observado, no que se refere à qualidade de uma IES, é a capacitação do seu corpo docente.

$\mathrm{Na}$ pesquisa realizada por Marchesan, Souza e Menezes (2011), foram identificadas três dimensões principais na avaliação que os discentes fizeram dos professores. A primeira é compromisso do professor em desenvolver no aluno as habilidades para a leitura, projetos de pesquisa e participação em eventos, sendo que essa última foi a que apresentou a média de desempenho mais baixa entre as dimensões e alta variabilidade relativa dos dados em torno da média. Na sequência, destacou-se, na pesquisa de Marchesan, Souza e Menezes (2011), relação entre os objetivos do curso e os conteúdos trabalhados e avaliados pelo professor e, por fim, preocupação do professor em estabelecer, em sala de aula, um ambiente que assegure um processo de ensino e aprendizagem de qualidade, a qual exibiu a maior média. Além disso, os autores ressaltam que a interdisciplinaridade não foi percebida pelos alunos como uma prática frequente.

Ainda a respeito dos docentes, Marks (2000) aponta a forte influência do relacionamento com o professor sobre a percepção de justiça nas avaliações e na aprendizagem percebida dos estudantes. Tem-se, ainda, que Alencar e Fleith (2004) realizaram uma pesquisa sobre práticas docentes e apresentam o professor como essencial no processo de desenvolvimento do aluno para adquirir competências que o levem a sua realização pessoal e profissional. Os autores igualmente destacam outros fatores que ajudam nesse desenvolvimento do aluno: os seus próprios interesses, o interesse no processo para dedicar-se e ter tempo e uma ampla bagagem de conhecimentos.

No tocante a esse papel do aluno em seu processo de aprendizagem, Marks (2000) ressalta que os estudantes mais comprometidos serão os que mais aprenderão. Ainda segundo o autor, um nível de exigência mais elevado, alinhado a conteúdo apropriado, proporcionará aprendizado, mas pode não proporcionar satisfação. Nesse sentido, Walter (2006) observou, em sua pesquisa, que a variável facilidade e flexibilidade do curso afeta negativamente a percepção da 
qualidade de ensino e da aprendizagem. A autora concluiu que, quanto menor o nível de exigência do curso, menor a percepção dos alunos em relação à sua aprendizagem e à qualidade do curso. Contudo, Marks (2000) alerta que, se os alunos considerarem que o nível de dificuldade e de exigência, bem como a carga de trabalho, forem injustos, haverá prejuízos na relação professor-aluno. Por isso, o autor propõe que os professores busquem um nível de equilíbrio entre carga de trabalho/nível de dificuldade e justiça nas avaliações.

Melo, Dutra e Oliveira (2001) observaram que três dos maiores gaps entre expectativa e percepção da qualidade dos alunos de administração por eles pesquisados encontraram-se em variáveis pautadas na relação entre a teoria e a prática: visitas de conferencistas; demonstração pelos professores de como o conteúdo teórico se aplica na prática; e orientação dos professores quanto à carreira profissional dos alunos. Isso indica que os alunos possuem uma expectativa elevada no que tange à integração entre teoria e prática. Além disso, segundo os autores, relacionar teoria e prática é importante para que os alunos percebam que o conhecimento abordado no curso poderá ser empregado em sua atuação profissional. Resultado similar obteve Walter (2006) em sua pesquisa, na qual identificou que a relação entre teoria e prática, assim como a autoeficácia e os métodos de ensino, influenciam a percepção dos alunos em relação à aprendizagem.

De forma geral, as dimensões mais evidenciadas na literatura foram, em síntese: percepção da aprendizagem, qualidade de ensino/desempenho do curso, satisfação geral do aluno, métodos de ensino, relação entre teoria e prática, facilidade e flexibilidade do curso, situação financeira do aluno, valor do curso, confiança nas pessoas da instituição, autoeficácia, infraestrutura, atitude da coordenação, grade curricular, atendimento dos funcionários da instituição, estágio, interdisciplinaridade, atitude dos professores, imagem social da instituição e compromisso emocional com a IES.

\section{DELINEAMENTO METODOLÓGICO}

Esta pesquisa, com abordagem quantitativa, parte integrante de um projeto mais amplo, caracteriza-se como descritiva, quanto aos objetivos, e de levantamento, quanto aos procedimentos. Segundo Hair Jr., Anderson e Tatham, (2005), a pesquisa descritiva visa à descrição de determinada situação e pode fazer uso da mensuração de um evento ou atividade. O levantamento, de acordo com Gil (2009), consiste na coleta de informações de um grupo significativo de pessoas, sendo que, a partir de análise quantitativa dessas informações, 
obtêm-se as conclusões. Como instrumento de coleta de dados quantitativos, utilizou-se o questionário estruturado, o qual foi obtido de Walter (2006). Por intermédio do questionário, se buscou identificar o desempenho de 26 dimensões, avaliadas por meio de 92 questões, para as quais cada respondente apontou seu nível de concordância utilizando uma escala intervalar que variou de 1 ("discordo totalmente") a 7 ("concordo totalmente"), bem como uma alternativa para "não sei avaliar". As 26 dimensões avaliadas foram: aprendizagem percebida (Apren); atendimento dos funcionários da instituição (AtFun); atitude da coordenação (AtCor); atitude dos professores (AtPro); atividades de estágio ou extracurriculares (ExCur); atualização dos conteúdos das disciplinas (AtuCo); autoeficácia dos alunos (AuEfi); auxílio financeiro (AuFin); auxílio pedagógico (AuPed); compromisso emocional dos alunos com a IES (CEmoc); confiança nas pessoas da IES (Confi); desempenho pessoal no curso (Dsemp); empregabilidade percebida oferecida pelo curso (Empre); grade curricular (Grade); imagem social da IES (ImagS); infraestrutura do campus (InCam); infraestrutura do curso (InCur); integração do aluno na vida acadêmica (Integ); métodos de ensino (MetEn); nível de exigência dos professores (Exig); organização das atividades do curso (Organ); qualidade de ensino/desempenho do curso (Quali); relação teoria e prática nas disciplinas (TxPra); satisfação geral com o curso (Satis); situação financeira do aluno (SitFi); e valor do curso (Valor).

Utilizou-se o questionário de Walter (2006) em virtude de sua abrangência, pois considerou os instrumentos de coleta de dados dos estudos de Hennig-Thurau, Langer e Hansen (2001), Alves (2003), Gonçalves Filho, Guerra e Moura (2003) e Marzo-Navarro, Pedraja-Iglesias e Rivera-Torres (2005) e foi adaptado à realidade brasileira por meio de grupo de foco.

Aplicou-se o instrumento de coleta de dados aos alunos de dois cursos de graduação em administração de uma IES do sul do país, sendo que, da população pesquisada, excluíram-se os primeiros e oitavos períodos, respectivamente, em decorrência de seu recente ingresso no curso e pelo fato de os alunos estarem em fase final do trabalho de conclusão de curso, não havendo necessidade de frequência diária em sala, o que poderia dificultar a aplicação. A pesquisa foi censitária, realizada com 25 turmas dos dois cursos pesquisados. Obtiveram-se 880 questionários respondidos, o correspondente a 76,19\% da população investigada, constituída pelos alunos presentes em sala de aula quando da aplicação da pesquisa.

Analisaram-se os dados por meio de métodos de estatística descritiva, análise fatorial, Alfa de Cronbach e regressão linear multivariada, utilizando, 
para isso, o software SPSS ${ }^{\text {TM }}$ 14.0. Após o tratamento dos dados, excluíram-se 133 casos com respostas inconsistentes ou inválidas, perfazendo 747 casos válidos. Neste estudo, efetuou-se a análise fatorial com o método de extração de componentes principais e método de rotação Varimax com normalização Kaiser. A análise fatorial tem como objetivo, segundo Maroco (2003), quantificar construtos que não podem ser observados diretamente. A redução do número de variáveis é efetuada pelo agrupamento de variáveis altamente correlacionadas convergindo em um fator; assim, um fator é constituído pela combinação linear de variáveis (DILLON; GOLDSTEIN, 1984). A análise fatorial tem o objetivo de comprovar as relações das variáveis indicadoras e seus possíveis agrupamentos em dimensões, ou seja, no caso desta pesquisa, em variáveis latentes (HAIR JR.; ANDERSON; TATHAM, 2005; MALHOTRA, 2001). Portanto, após o tratamento dos dados, efetuou-se uma análise fatorial para re-especificar as dimensões estudadas. Também foi empregado o Alfa de Cronbach para analisar a confiabilidade das dimensões geradas a partir da análise fatorial (HAIR JR.; ANDERSON; TATHAM, 2005). Em seguida, para verificar a influência dos diferentes atributos na aprendizagem percebida, optou-se por realizar a regressão linear multivariada que, de acordo com Maroco (2003), é utilizada para identificar as relações entre variáveis e predizer a influência de um conjunto de variáveis independentes em uma variável dependente. O método de cálculo selecionado foi Enter, que inclui todas as variáveis, sejam elas significativas ou não (FERREIRA, 1999).

Calculou-se o modelo de regressão linear, apresentado na Equação 1, com base em Maroco (2003).

Equação 1: $Y j=\beta_{0}+\beta_{1} X_{1} j+\beta_{2} X_{2}+\ldots+\beta p X p j+E j(j=1, \ldots, n)$

\section{APRESENTAÇÃO E ANÁLISE DOS RESULTADOS}

A Tabela 1 apresenta, simultaneamente, os resultados da análise fatorial e do Alfa de Cronbach, tendo sido esse último realizado apenas com as 16 dimensões utilizadas após o agrupamento das variáveis. Utilizou-se o Alfa de Cronbach para analisar a consistência interna dos resultados de cada variável latente e a confiabilidade do instrumento.

Segundo Hair Jr., Anderson e Tatham, (2005), a análise fatorial fornece uma visão muito direta das inter-relações entre variáveis e desempenha um importante papel complementar por meio de resumo e redução de dados. Sob a perspectiva do resumo de dados, a análise fatorial fornece uma clara 
compreensão sobre quais variáveis podem atuar juntas e quantas variáveis podem ser consideradas com impacto na análise. Assim, a análise fatorial fornece a base para a criação de um novo conjunto de variáveis que incorpore o caráter e a natureza das variáveis originais em um número muito menor de novas variáveis.

O resultado da análise fatorial, conforme descrito na Tabela 1, resultou em 15 dimensões, usando como critério de retenção do fator o critério da raiz latente (autovalor da dimensão maior que a unidade). Contudo, optou-se por manter as dimensões "Quali" e "Satis" separadas da dimensão 1 - "Apren" -, em virtude de as dimensões qualidade de ensino (HENNIG-THURAU; LANGER; HANSEN, 2001; LIN; TSAI, 2006; WALTER, 2006) e satisfação (ALVES, 2003; GONÇALVES FILHO; GUERRA; MOURA, 2003; WALTER, 2006) serem reconhecidas na revisão de literatura como dimensões distintas e influenciadoras da aprendizagem.

A partir do resultado do Alfa de Cronbach, a dimensão "ExCur" (atividades de estágio ou atividades extracurriculares) foi excluída em virtude do baixo índice obtido. O limite inferior aceito para o Alfa de Cronbach é de 0,70, podendo diminuir para 0,60 em pesquisas exploratórias (HAIR JR.; ANDERSON; TATHAM, 2005; MALHOTRA, 2001). Um coeficiente Alfa de Cronbach maior que 0,70 demonstra um grau de fidelidade aceitável (HAIR JR.; ANDERSON; TATHAM, 2005). Esses índices altos do Alfa de Cronbach revelam a validade das variáveis indicadoras em relação às suas variáveis latentes.

Dado o exposto, teve-se como resultado 16 dimensões analisadas e, para fins de regressão linear, utilizou-se o escore fatorial em cada uma das dimensões sendo elas: percepção da aprendizagem (Apren), como variável dependente, e, como variáveis independentes: qualidade de ensino/desempenho do curso (Quali), satisfação geral do aluno (Satis), métodos de ensino (MetEn), facilidade e flexibilidade do curso (Facil), valor do curso e situação financeira (ValFin), confiança nas pessoas da instituição (Confi), autoeficácia (AuEfi), infraestrutura (Infra), atitude da coordenação (AtCor), grade curricular (Grade), empregabilidade do curso (Empre), atendimento dos funcionários da instituição (AtFun), imagem social da instituição (ImagS), auxílio pedagógico (AuPed) e integração na vida acadêmica (Integ).

A Tabela 2 apresenta os coeficientes de adequação da regressão linear. 


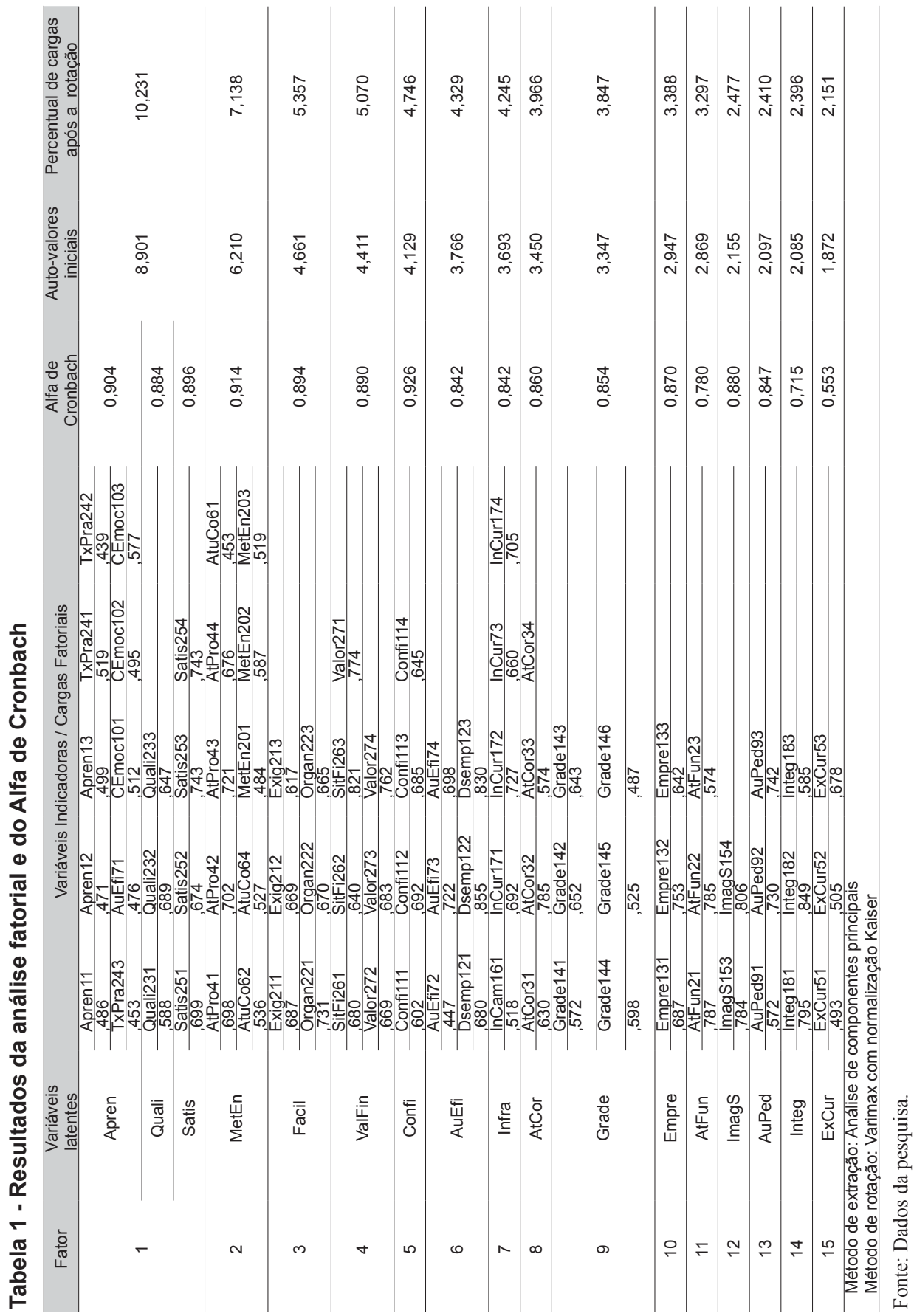



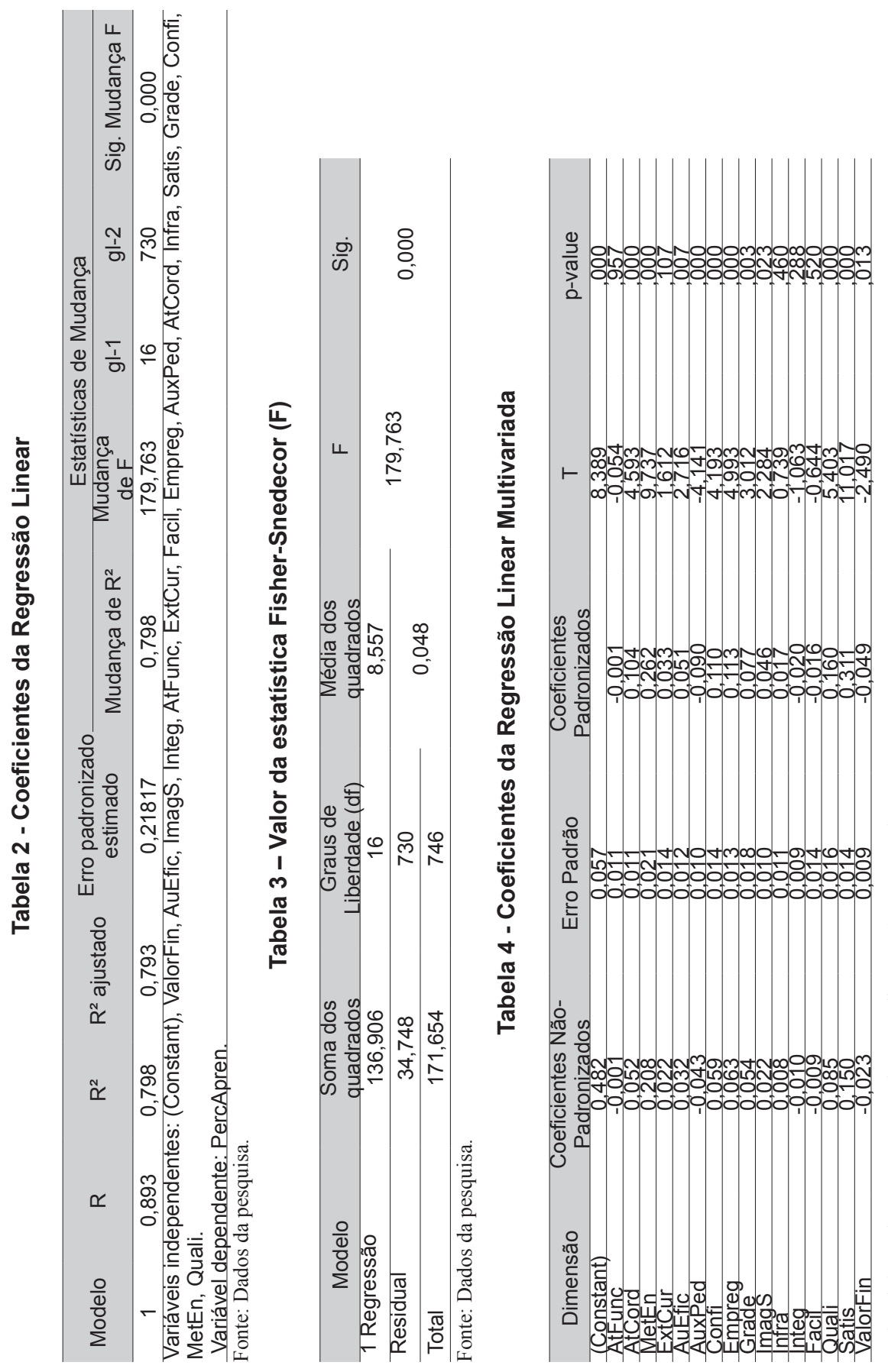
Conforme se pode observar na Tabela 2, o coeficiente de determinação $\left(\mathrm{R}^{2}\right.$ ajustado) estima que $79,3 \%$ da variabilidade total da aprendizagem percebida pelos alunos da instituição é explicada pelas variáveis independentes no modelo de regressão linear ajustado. A Tabela 3 complementa a análise indicando o valor da estatística Fisher-Snedecor (F) que, com um valor $\mathrm{p}<0,000$, rejeita fortemente a hipótese nula de ausência de regressão.

A Tabela 4 apresenta os coeficientes da regressão que tiveram como variável dependente a aprendizagem percebida dos alunos.

Por meio dos resultados da Tabela 4, podem-se indicar os resultados do modelo da regressão linear representado na Equação 2:

Equação 2: PercAprend = 0, 482 (Constant) + 0,311 (Satis) + 0,262 (MetEn $)+0,160$ (Quali) + 0,113 (Empreg) + 0,110 (Confi) + 0,104 (AtCord) + 0,077 (Grade) + 0,051 (AuEfic) + 0,046 (ImagS) - 0,049 (ValorFin) - 0,090 (AuxPed).

A análise dos valores absolutos dos coeficientes da regressão padronizados permite concluir que as variáveis que mais afetam significativamente a aprendizagem percebida dos alunos da IES analisada são satisfação $(0,311)$, métodos de ensino $(0,262)$ e qualidade $(0,160)$.

A percepção da aprendizagem, variável dependente, refere-se ao aprendizado, à aplicabilidade à vida profissional e ao desenvolvimento pessoal. Já a variável independente satisfação foi mensurada no que tange à satisfação em relação à escolha da instituição, à escolha do curso, atendimento das expectativas relacionadas ao curso e satisfação geral com o curso. Métodos de ensino, segunda dimensão que mais afeta a aprendizagem percebida, constitui-se por clareza na exposição dos temas, domínio do conteúdo pelos professores e adequação dos métodos de ensino. Nesse sentido, Walter (2006) já havia identificado que métodos de ensino influenciam a percepção dos alunos em relação à aprendizagem. Quanto à qualidade, terceira dimensão, foi avaliada por meio das variáveis indicadoras: qualidade melhor do que a da concorrência, atendimento das necessidades dos alunos e qualidade geral do curso.

Com uma influência um pouco menor, encontram-se as dimensões: empregabilidade $(0,113)$, confiança nas pessoas da instituição $(0,110)$, atitude da coordenação $(0,104)$, grade curricular $(0,077)$, autoeficácia $(0,051)$ e imagem social $(0,046)$.

A empregabilidade refere-se à perspectiva de inserção no mercado de trabalho, à melhoria de oportunidades no mercado de trabalho para alunos formados 
na instituição e à possibilidade de promoções no emprego proporcionadas pelo curso. A confiança nas pessoas da instituição está relacionada ao atendimento dos interesses dos alunos pela instituição, à confiança nos funcionários da universidade, à integridade dos funcionários da universidade e ao cumprimento de promessas. No que concerne aos funcionários da instituição, Melo, Dutra e Oliveira (2001) destacam que o desempenho desses influencia a percepção que os estudantes possuem a respeito da qualidade do ensino. A atitude da coordenação diz respeito à facilidade de acesso à coordenação, ao apoio da coordenação em atividades desenvolvidas pelos alunos, ao feedback das reclamações e às sugestões dos alunos e resolução de problemas pela coordenação. A grade curricular foi avaliada por meio da adequação da carga horária das disciplinas, ordenação das disciplinas na grade curricular, existência de disciplinas optativas, direcionamento do curso para o mercado de trabalho, qualidade do conteúdo das disciplinas e relevância das disciplinas para a profissão. Karemera, Reuben e Sillah (2003) também verificaram que a escolha das matérias está associada significativamente ao desempenho dos alunos. A autoeficácia refletiu o interesse do aluno pelo curso, adequação de formação prévia ao curso, satisfação com desempenho pessoal e relação da adequação do desempenho com os objetivos pessoais. Essa variável também foi identificada por Walter (2006) como fator que influencia a aprendizagem percebida. A imagem social da instituição foi avaliada por meio do caráter inovador e visão de futuro da instituição, confiança na instituição, contribuição da instituição para a sociedade e envolvimento da universidade na comunidade.

Para avaliar a dimensão valor financeiro $(-0,049)$, levou-se em consideração a relação entre o investimento no curso e possibilidades de emprego, a adequação da relação preço versus qualidade do curso, a compatibilidade entre custo versus benefício e a justiça do valor da mensalidade. Já para o auxílio pedagógico (-0,090), consideraram-se os itens: a instituição disponibiliza acompanhamento extraclasse aos alunos, a instituição disponibiliza atendimento dos professores aos alunos em horários extraclasse e a disposição dos professores para atendimento extraclasse. As dimensões valor financeiro e auxílio pedagógico apresentaram, respectivamente, os valores $-0,049$ e $-0,090$ respectivamente. A presença de coeficientes com valor negativo para esses construtos encontra-se em oposição aos pressupostos teóricos de que o valor financeiro e o auxílio pedagógico devam influenciar positivamente a percepção da aprendizagem, ensejando pesquisas futuras para aprofundar esta questão.

A Tabela 5 apresenta a análise dos resíduos do modelo de regressão, corroborando a adequação do modelo proposto aos dados analisados. 
Tabela 5 - Resíduos do modelo de regressão

\begin{tabular}{lccccc}
\hline & Mínimo & Máximo & Média & Desvio Padrão & $\mathbf{N}$ \\
\hline Valor Previsto & 1,0380 & 3,5624 & 2,6329 & 0,42839 & 747 \\
\hline Resíduo & $-0,6436$ & 0,68922 & 0 & 0,21582 & 747 \\
\hline Valor previsto padronizado & $-3,7230$ & 2,170 & 0 & 1 & 747 \\
\hline Resíduo Padronizado & $-2,950$ & 3,159 & 0 & 0,989 & 747 \\
\hline
\end{tabular}

Fonte: Dados da pesquisa.

Por meio da Tabela 5 visualiza-se a estatística descritiva dos resíduos da regressão. O número de casos $(\mathrm{N})$ foi de 747.

A Figura 1 ilustra a distribuição dos resíduos que é complementada pelo teste de normalidade apresentado na Tabela 6.

Figura 1 - Residual padronizado do modelo de regressão

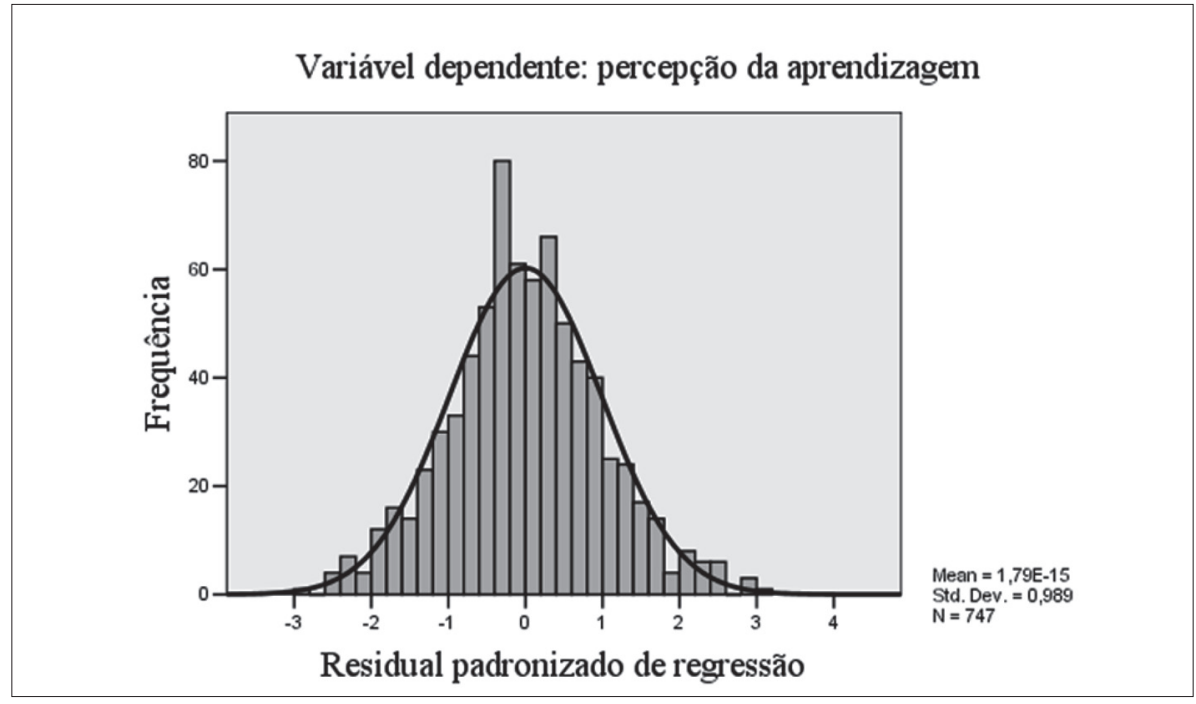

Fonte: Dados da pesquisa.

A Tabela 6 apresenta o teste de normalidade dos resíduos da regressão, o qual pressupõe que Ho: resíduos apresentam distribuição normal. Sempre que os resíduos são padronizados, a média (Mean) destes fica próxima de zero e o desvio padrão (Std. Dev.) de 1,0. 
Tabela 6 - Teste de normalidade dos resíduos da regressão

\begin{tabular}{ccccccc}
\hline \multicolumn{3}{c}{ Kolmogorov-Smirnov(a) } & \multicolumn{2}{c}{ Shapiro-Wilk } \\
\hline \multirow{2}{*}{$\begin{array}{c}\text { Resíduos } \\
\text { padronizados }\end{array}$} & \begin{tabular}{c} 
Estatística \\
\cline { 3 - 7 }
\end{tabular} & $\begin{array}{c}\text { Grau de } \\
\text { liberdade (df) }\end{array}$ & Significância & Estatística & $\begin{array}{c}\text { Grau de } \\
\text { liberdade (df) }\end{array}$ & Significância \\
\cline { 2 - 8 } & 0,024 & 747 & 0,200 & 0,997 & 747 & 0,099 \\
\hline
\end{tabular}

Fonte: Dados da pesquisa.

Por meio da Tabela 6, observa-se que não há evidências para rejeitar a hipótese nula de normalidade dos resíduos, pois o valor de $\mathrm{p}$ foi não-significante, tanto para o teste de Kolmogorov-Smirnov, quanto para o teste de ShapiroWilk. Conforme Maroco (2003), quando a análise dos resíduos indica a sua normalidade, o modelo de regressão é válido.

\section{CONSIDERAÇÕES FINAIS}

Esta pesquisa teve por objetivo analisar as dimensões que influenciam a aprendizagem dos alunos dos cursos de graduação em administração de uma IES no sul do Brasil. Por meio da revisão de literatura e da análise fatorial, encontraram-se 15 dimensões que indicam relação com a aprendizagem.

Por meio do coeficiente de determinação $\left(\mathrm{R}^{2}\right)$, verificou-se que $79,3 \%$ da variabilidade total da aprendizagem percebida pelos alunos da instituição pode ser explicada pelas variáveis independentes no modelo de regressão linear ajustado. Dessa forma, conclui-se que a percepção da aprendizagem é influenciada, principalmente, pelas dimensões satisfação, métodos de ensino e qualidade do ensino, as quais apresentaram fortes evidências de influência na variável dependente aprendizagem percebida $(\mathrm{p}<0,000)$. Esse resultado indica que a percepção da aprendizagem dos alunos envolve fatores que se relacionaram à satisfação em relação à instituição, à escolha do curso e ao atendimento das expectativas referentes ao curso, bem como quanto à satisfação geral com o curso. Tendo em vista os métodos de ensino, os alunos consideram importante a clareza na exposição dos temas, o domínio do conteúdo pelos professores e a adequação dos métodos de ensino para aplicação. Têm-se, ainda, as variáveis indicadoras: qualidade melhor do que a da concorrência, atendimento às necessidades dos alunos e qualidade geral do curso com adequada significância para rejeitar a hipótese nula de não influência na variável dependente $(\mathrm{p}<0,05)$.

As dimensões apontadas pelos alunos podem, como contribuição prática, auxiliar a IES a direcionar suas ações de forma a realçar os itens considerados importantes pelos alunos, principalmente no que tange à satisfação, aos méto- 
dos de ensino e à qualidade do curso. Como contribuição teórica para a área de estudos, acredita-se que esta pesquisa possa auxiliar, por meio do número abrangente de dimensões, no entendimento de fatores que possam influenciar a aprendizagem de estudantes de cursos de graduação em administração. Isso porque, na literatura, não se identificaram estudos que abordassem, especificamente, a aprendizagem percebida, com destaque para a influência que essa sofre de outras dimensões avaliadas pelos alunos do Ensino Superior.

Como limitação desta pesquisa, aponta-se que os resultados estão associados à visão de alunos, e não da sociedade, podendo o aluno ter uma visão momentânea e distorcida. Outra limitação é que a presente pesquisa não pode ser generalizada, pois foca uma situação momentânea dos cursos de administração analisados. Estudos futuros poderiam testar essas e outras variáveis que possam influenciar a aprendizagem percebida de alunos de outras instituições de ensino. Outras abordagens metodológicas para o tema também podem ser úteis para aprofundar a compreensão do assunto, como por exemplo, modelos não-lineares (KANO, 1984).

\section{REFERÊNCIAS}

ALENCAR, Eunice M.; FLEITH, Denise de S. Inventario de praticas docentes que favorecem a criatividade no ensino superior. Psicologia: Reflexão e Crítica, Porto Alegre, v. 17, n. 1, p. 105-110, 2004.

\section{ALVES, Helena M. B. Uma abordagem de marketing à satisfação do aluno no ensino universitário público: índice, antecedentes e} consequências. 2003. 285f. Tese (Doutorado em Gestão) - Departamento de Gestão e Economia, Universidade da Beira Interior, Covilhã, Portugal, 2003.

DILLON, William R.; GOLDSTEIN, Matthew. Multivariate Analysis. New York: John Wiley \& Sons, 1984.

FERREIRA, Armando M. SPSS - Manual de utilização. Escola Superior Agrária de Castelo Branco, 1999.

GARCIA, Mauro N.; DERISIO, Diana P. Fatores de qualidade percebidos pelos discentes do curso de administração de IES municipais do Grande ABC. Revista de Administração da UNIMEP, Piracicaba, v. 5, n. 2, p. 1-27, maio/ago. 2007. 
GIL, Antonio C. Métodos e técnicas de pesquisa social. 6. ed. São Paulo: Atlas, 2009.

GONÇALVES FILHO, Cid; GUERRA, Renata S.; MOURA, Alexandre. Mensuração de satisfação, qualidade, lealdade com a instituição, valor e expectativa em instituições de ensino superior: um estudo do modelo ACSI através de equações estruturais. In: ENCONTRO DA ASSOCIAÇÃO NACIONAL DOS PROGRAMAS DE PÓS-GRADUAÇÃO EM ADMINISTRAÇÃO, 27., 2003, Atibaia, SP. Anais... Rio de Janeiro: ANPAD, 2003.

HAIR JR., Joseph F.; ANDERSON, Rolph E.; TATHAM, Ronald L. Análise multivariada de dados. 5. ed. Porto Alegre : Bookman, 2005.

HENNIG-THURAU, Thorsten; LANGER, Markus F.; HANSEN, Ursula. Modeling and managing student loyalty: an approach based on the concept of relationship quality. Journal of Service Research, Thousand Oaks, v. 3, n. 4 , p. 331-344, may. 2001.

KAREMERA, David; REUBEN, Lucy J.; SILLAH, Marion R. The effects of academic environment and background characteristics on student satisfaction and performance: the case of South Carolina State university's school of business. College Student Journal, Chula Vista (CA), v. 37, n. 2, p. 298-308, 2003.

KANO, N., Attradive. Quality and Must-be Quality. Society for Quality Control, Tokyo, p.39-48, abril, 1984.

LIN, Chieh-Peng; TSAI, Yuan H. Modeling educational quality and student loyalty: a quantitative approach based on the theory of information cascades. Quality \& Quantity, Itália, v. 42, n. 3, p. 397-415, 2006.

MALHOTRA, Naresh K. Pesquisa de marketing: uma orientação aplicada. 3. ed. Porto Alegre: Bookman, 2001.

MARCHESAN, Teresinha M.; SOUZA, Adriano M.; MENEZES, Rui. Avaliação do Processo de ensino: uma Abordagem multivariada. Produção, São Paulo, v. 21, n. 2, abr./jun. 2011.

MARKS, Ronald B. Determinants of student evaluations of global measures of instructor and course value. Journal of Marketing Education, Thousand Oaks (CA), v. 22, n. 2, p. 108-119, aug. 2000. 
MAROCO, João. Análise estatística. 2. ed. Lisboa : Edições Silabo, 2003.

MARZO-NAVARRO, Mercedes; PEDRAJA-IGLESIAS, Marta; RIVERATORRES, M. Pilar. Measuring customer satisfaction in summer courses. Quality Assurance in Education, Bradford (UK), v. 13, n. 1, p. 56-65, 2005.

MELLO, Sérgio C. B. de; DUTRA, Hérrisson F. de O.; OLIVEIRA, Patrícia A. da S. Avaliando a qualidade de serviço educacional numa IES: o impacto da qualidade percebida na apreciação do aluno de graduação. Organizações \& Sociedade, Salvador, v. 8, n. 21, p. 125-137, maio./ago. 2001.

REINERT, José N.; REINERT, Clio. Estudante não é cliente: é parceiro. In: ENCONTRO DA ASSOCIAÇÃO NACIONAL DE PÓS-GRADUAÇÃO E PESQUISA EM ADMINISTRAÇÃO, 29., 2005, Brasília, DF. Anais... Rio de Janeiro: ANPAD, 2005.

RODIE, Amy R.; KLEINE, Susan S. Customer participation in services production and delivery. In: SWARTZ, Teresa A; IACOBUCCI, Dawn (Eds.). Handbook of service marketing and management, Sage: Thousand, p. 111-125, 2000.

SOUZA, Saulo A. de; REINERT, José N. Avaliação de um curso de ensino superior através da satisfação/insatisfação discente. Avaliação, Campinas; Sorocaba, v. 15, n. 1, p. 159-176, mar. 2010.

WALTER, Silvana A. Antecedentes da satisfação e da lealdade de alunos de uma instituição de ensino superior. 2006. 167f. Dissertação (Mestrado em Administração) - Programa de Pós-Graduação em Administração, Universidade Regional de Blumenau, Blumenau, 2006.

WALTER, Silvana A.; TONTINI, Gérson; DOMINGUES, M. J. C. S. Análise da Satisfação do Aluno para Melhoria de um Curso de Administração. Revista FACES, Belo Horizonte, v. 6, p. 52-70, 2006. 